\title{
Heat Transfer in Black Holes
}

\author{
R.L. Corral-Bustamante ${ }^{a}$, D. Sáenz, N.I. Arana, D. Cárdenas \\ Department of Industrial \& Mechatronics Engineering , Technological Institute of Cuauhtémoc City, \\ Tecnológico Ave. S/N, Z.P. 31500, Cuauhtémoc City, Chihuahua, México \\ aleticia.corral@cimav.edu.mx
}

Keywords: Heat transfer, black hole, event horizon, entropy, quantized energy, gigantic mass, dark material collapsed, space-time continuum, metric, relativistic equations

\begin{abstract}
In a universe in indefinite expansion, undetermined amounts of dark material suspended in the space-time continuum exist. The case of 'sufficient' amount of material contracted throughout time due to its density can occur. Using relativistic equations, in the present work a thermodynamic system was considered whose volume of control is limited by a gigantic mass that is suspended in space in order to predict its behavior. Given the obtained results, evidence of a certain quantized dark material in process of collapse or collapsed into a black hole can be argued.

The heat transfer of the black hole was measure through entropy change with respect to the time and other forms of energy, it was determined that the hole emits particles to surroundings from its event horizon (black hole 'shining'). The emissions are proportional to the disappearance of the hole; they are candidates to integrate the "baby universes" of Hawking with new histories that satisfy the theorem "a black hole has no hair".
\end{abstract}

\section{Introduction}

Solutions of the relativistic equations that give place to singularities [1] in real time and/or in imaginary time, allow predicting a behavior of masses in space-time. Here the universe is a gigantic mass bordered by a volume of control in a thermodynamic system [2,3]. The simulation of the model obtained by means of relativistic equations [4-9], throws data of something similar to dark material that is scattered in the universe and confined in "baby universes" [10] probably constituent of black holes, white dwarfs or neutrinos stars, such as the particles of the gigantic mass were contracted to a field of enormous gravity [10-12]. The baby universes, that take the particles that fell into the hole, occur in what is called, imaginary time.

Also, the obtained results allow predicting a closed link between the black holes and thermodynamic science by means of predictions of the entropy that presents the black hole when it emits particles from its event horizon [13]. The heat transfer in black holes measured through the entropy with respect to other forms of energy that take place during their emissions, is the responsible of the new histories of the particles thrown towards the surroundings. The solution obtained for the postulated metric give a place to imaginary numbers that allow glimpsing material with quantized energy. The theorem "a black hole has no hair" [14-21], concludes that the behavior of the gigantic mass compressed to a black hole with the mass of a mountain, is conceived by the quantum mechanics like a microscopic radius particle smaller than the radius of a neutron or a proton, which allows to demonstrate an intrinsic bond between General Relativity and the Quantum Theory through the Quantum Gravity.

\section{Nomenclature}

$\begin{array}{ll}{ }_{-} C n, c_{n} & \text { Constants to be determined } \\ E & \text { Energies: kinetic, potential, internal, quantized, }[\mathrm{kJ}] \\ G & \text { Universal Gravitation Constant, } G=6.67\left(10^{-8}\right),\left[\text { dyne } \mathrm{cm}^{2} / \mathrm{g}^{2}\right] \\ G_{i j}=R_{j k l}^{i} & \text { Riemann-Christoffel tensor or contracted tensor of Einstein } \\ h & \text { Constant for a system of mass, }\left\lfloor\mathrm{cm}^{2} / \mathrm{s}\right\rfloor\end{array}$




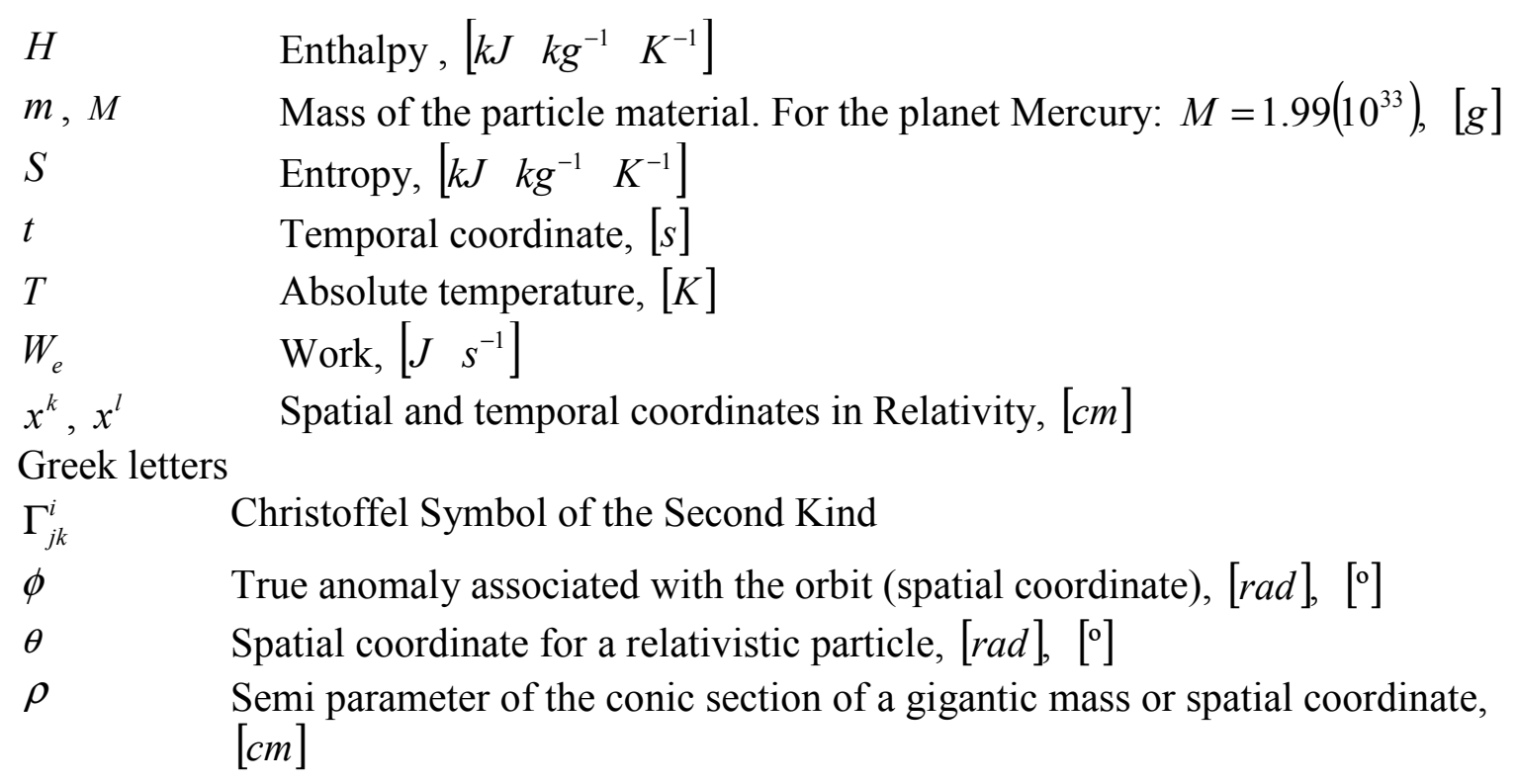

\section{Modelling}

In this work, a metric in energy terms in coordinates in a 4-dimensional space is postulated:

$\left(x^{1}, x^{2}, x^{3}, x^{4}\right)=\left(m S T, m H, E, W_{e} t\right)$ or $\left(x^{1}, x^{2}, x^{3}, x^{4}\right)=(S, H, E, t)$, corresponding to a metric based in quasi-spherical coordinates: $\left(x^{1}, x^{2}, x^{3}, x^{4}\right)=(\rho, \theta, \phi, t)[4]$

$g_{11}=-\cos (u) ; g_{22}=-S^{2} ; \quad g_{33}=-S^{2} \sin ^{2}(H) ; g_{44}=\cos (v)$

where $u_{\mathrm{y}} v$ are functions of $S$. For this metric, Eq. 1, the non-zero components of Christoffel symbols of the second kind are given by:

$$
\begin{aligned}
& \{1,11\}=-\sin (u) \partial u / \partial S / 2 \cos (u),\{1,22\}=-S / \cos (u),\{1,33\}=-S \sin ^{2}(H) / \cos (u),\{2,12\}=\{3,13\}=1 / S \\
& \{3,23\}=\cos (H) / \sin (H),\{1,44\}=-\sin (v) \partial v / \partial S / 2 \cos (u),\{2,33\}=-\sin (H) \cos (H), \\
& \{4,14\}=-\sin (v) \partial v / \partial S / 2 \cos (v) .
\end{aligned}
$$

From Eqs. 2 non-zero components of the Einstein tensor ${ }^{1}$ are computed as:

$$
\begin{aligned}
& G_{11}=\frac{\cos (u) \cos (v)-\cos (v)+S \sin (v)(\partial v / \partial S)}{S^{2} \cos (v)}, \quad \begin{array}{l}
G_{22}=\frac{S}{4} \frac{2 \sin (u)(\partial u / \partial S) \cos ^{2}(v)}{\cos ^{2}(u) \cos ^{2}(v)}+ \\
G_{33}=\frac{S \sin ^{2}(H)}{4}-\frac{2 \sin (u)(\partial u / \partial S) \cos ^{2}(v)}{\cos ^{2}(u) \cos ^{2}(v)} \\
+\frac{2 \sin (v)(\partial v / \partial S) \cos (u) \cos (v)+\ldots+\ldots+\ldots}{\cos ^{2}(u) \cos ^{2}(v)},
\end{array} G_{44}=\frac{\cos (v)\left(S \sin (u)(\partial u / \partial S)-\cos ^{2}(u)+\cos (u)\right)}{S^{2} \cos ^{2}(u)}
\end{aligned}
$$

From the solution of the Einstein tensor, Eq. 3, it is obtained:

$$
{ }^{1} G_{i j}=R_{j k l}^{i} \equiv \frac{\partial \Gamma_{j l}^{i}}{\partial x^{k}}-\frac{\partial \Gamma_{j k}^{i}}{\partial x^{l}}+\Gamma_{j l}^{r} \Gamma_{r k}^{i}-\Gamma_{j k}^{r} \Gamma_{r l}^{i}
$$


$u(\rho)=\arccos \left(\left(\left(-_{-C 1-_{-} C 2 \rho}^{\rho}+\left(-\frac{-C 2}{\rho}-\frac{-C 1-_{-} C 2 \rho}{\rho^{2}}\right) \rho\right) \rho /\left({ }_{-} C 1-_{-} C 2 \rho\right)\right)\right)$.

$v(\rho)=\arccos \left(\frac{-C 1-_{-} C 2 \rho}{\rho}\right)$.

where $\rho=S$. Thus the metric for the calculation of the geodesic is given by $g_{11}=u(\rho), g_{44}=v(\rho)$. In this way, the geodesics are:

$$
\begin{aligned}
& S_{s, s}+\frac{1}{2} \frac{{ }_{-} C 1 S_{s}^{2}}{S\left(\_C 1-_{-} C 2 S\right)}+\frac{\left(\_C 1-_{-} C 2 S\right) H_{s}^{2}}{{ }_{-} C 2}+ \\
& \frac{\left({ }_{-} C 1-_{-} C 2 S\right) \sin ^{2}(H) E_{s}^{2}}{-C 2}+\frac{C 1-\left({ }_{-} C 2 S\right)_{-} C 1 t_{s}^{2}}{2 S^{3}{ }_{-} C 2}=0, \quad E_{s, s}+2 S_{s} E_{s} / S+2 \cos (H) H_{s} E_{s} / \sin (H)=0 \\
& H_{s, s}+2 S_{s} H_{s} / S-\sin (H) \cos (H) E_{s}^{2}=0, t_{s, s}{ }_{-} C 1 S_{s} t_{s} / S\left({ }_{-} C 1-_{-} C 2 S\right)=0 \text {. }
\end{aligned}
$$

The relativistic equation obtained from the geodesic solution, Eq. 6, is:

$$
\begin{aligned}
& { }_{-} C 2^{2}\left(e^{-2}\right)^{2} \frac{d^{2} S}{d E^{2}}+\frac{1}{2}=\frac{C 11_{-} C 2^{2}\left(e^{-2}\right)^{2} d S / d E}{S\left({ }_{-} C 1-_{-} C 2 S\right)} \\
& +\frac{1}{2} \frac{S_{-} C 2\left(1_{-} C 4^{2}\right.}{\left.S 1-\_C 2 S\right)}=0
\end{aligned}
$$

where, $S=1 / u$. A specific form is given to Eq. 7, then, it is compared with the Newtonian equation for planetary motion:

$$
\left(\frac{\partial u}{\partial \phi}\right)^{2}+u^{2}-\frac{2 G M u}{h^{2}}+\frac{-C 5}{h^{2}}=0
$$

In order to obtain constants value $C 1,{ }_{-} C 2,{ }_{-} C 4$, and $\_C 5$. The relativistic equation solution is given in real and imaginary terms; it has the incomplete elliptic integral of the first kind, EllipticF

$$
\begin{aligned}
& E=\phi=c_{1} I \times \\
& \frac{\left[\begin{array}{l}
c_{2} \sqrt{I\left(-c_{3} \rho-c_{4}-c_{5} I\right)} \sqrt{\left(c_{6}-c_{7} I\right)\left(-c_{8} \rho+c_{9}\right)} \sqrt{I\left(c_{3} \rho+c_{4}-c_{5} I\right)} \times \\
\text { EllipticF }\left(c_{10} \sqrt{I\left(-c_{3} \rho-c_{4}-c_{5} I\right)}, c_{11}+c_{12} I\right) \sqrt{c_{13}^{3}+c_{14}^{2}+c_{15}-c_{16}} \\
-c_{17} \sqrt{I\left(-c_{3}-c_{4}-c_{5} I\right)} \sqrt{\left(c_{6}-c_{7} I\right)\left(-c_{8}+c_{9}\right)} \sqrt{I\left(c_{3}+c_{4}-c_{5} I\right)} \times \\
\text { EllipticF }\left(c_{10} \sqrt{I\left(-c_{3}-c_{4}-c_{5} I\right)}, c_{11}+c_{12} I\right) \sqrt{c_{13} \rho^{3}+c_{14} \rho^{2}+c_{15} \rho-c_{16}}
\end{array}\right]}{\sqrt{c_{13} \rho^{3}+c_{14} \rho^{2}+c_{15} \rho-c_{16}} \sqrt{c_{13}^{3}+c_{14}^{2}+c_{15}-c_{16}}} .
\end{aligned}
$$

\section{Results and Discussion}

The obtained results from the proposed metric, are given through measurements of entropy, $S$, and their speed of change, and/or, the semi-parameter of the associated conic section with the orbit of 
the gigantic mass, $\rho$, that modeled, gave place to dark material which probably is the one that constitutes a black hole or other celestial bodies.

In Table 1 is shown that the ranks in which the dependent variable was plotted ( $\rho$ or $S$ ) in Figs. 13 are "too large" with respect to the ranks of the abscissae $(\operatorname{Re}(S)$ or $\operatorname{Re}(\rho))$ and the ordinates $(\operatorname{Im}(S)$ or $\operatorname{Im}(\rho))$ that the observer can appreciate and that are product of the simulation of the proposed metric for the prediction of the material's behavior.
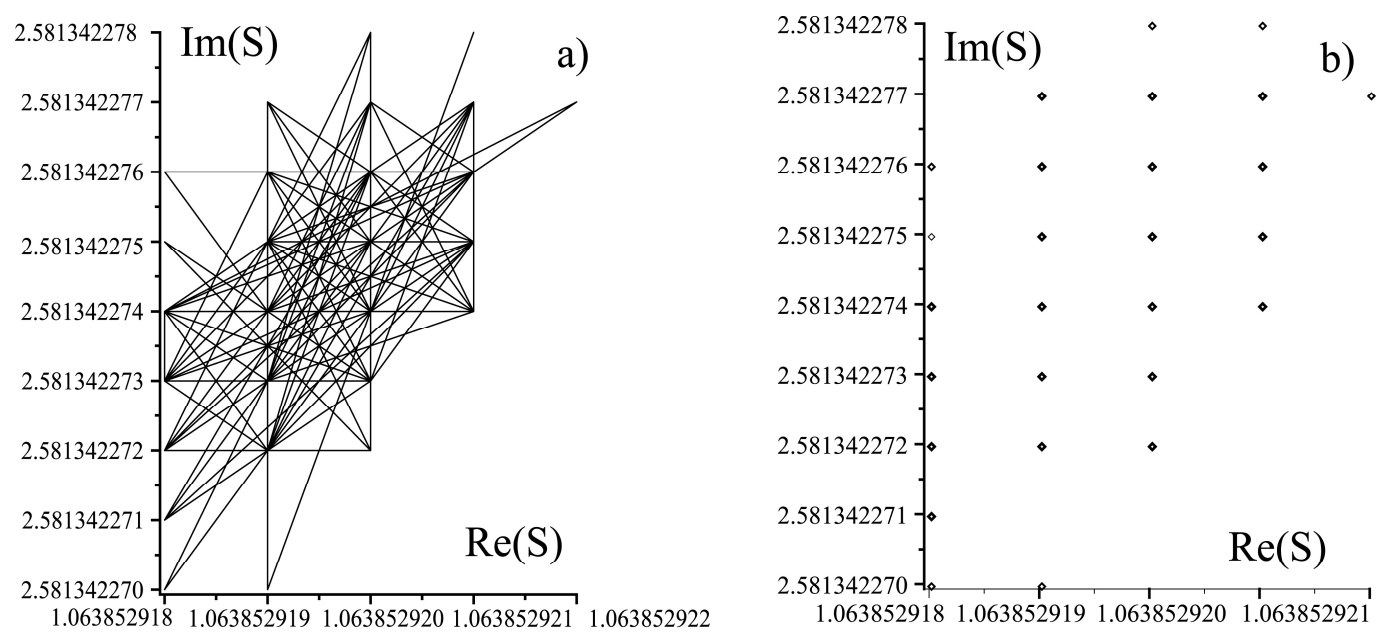

Fig. 1 Dark material of a black hole, a white dwarf or a neutron star, suspended in a universe without boundaries or infinite, confined in a small universe in imaginary time.

a) Quantity of entropy contained in this small region. b) Energy quantized.
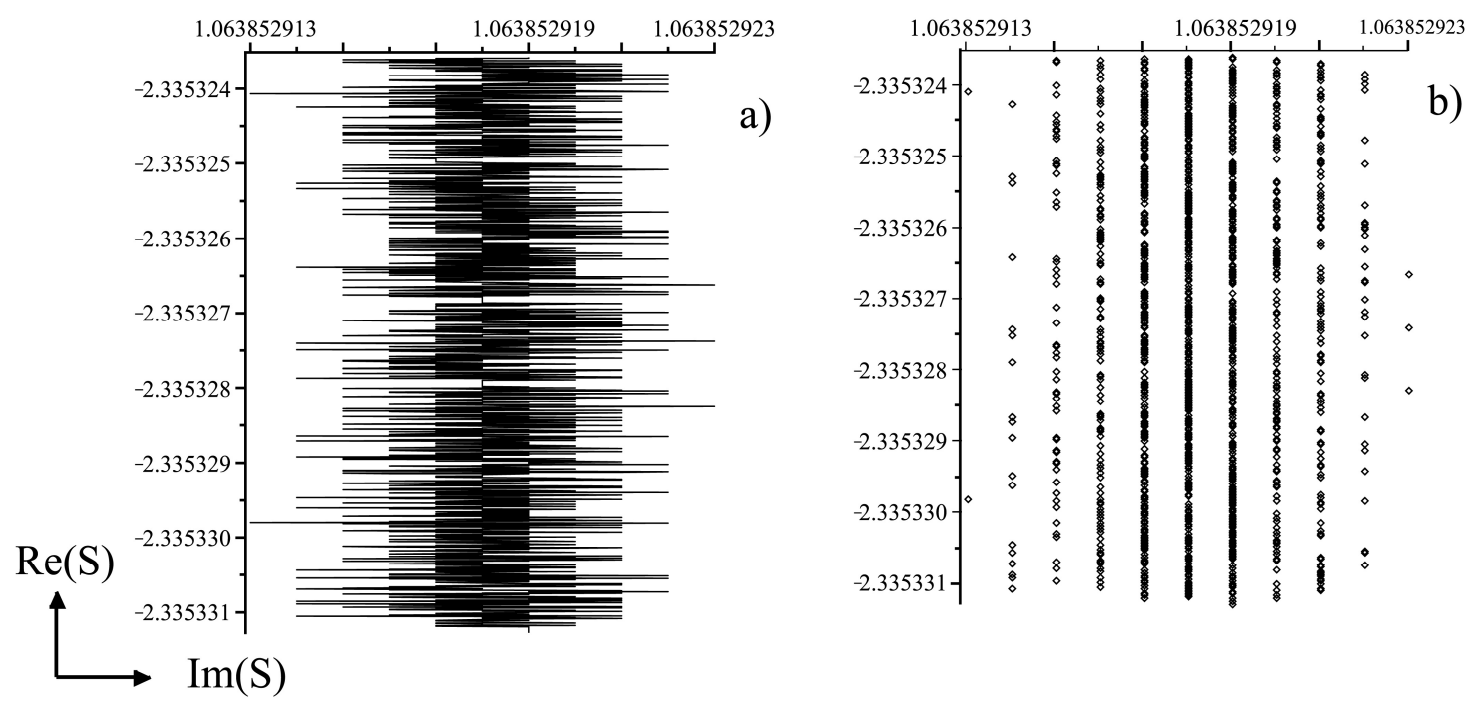

Fig. 2 Behavior of a gigantic mass collapsed to a black hole in continuum space-time:

a) Entropy, b) Energy quantized; beginning and end of the black hole.

The ranks in which the observer can appreciate more information correspond to Fig 3 . This allows concluding that the graphs obtained through Figs. 1-3 seem to resemble particle histories with certain properties that are developed in imaginary time, instead of real time in which we perceived the existence. This material is responsible of formation of Hawking's "baby universes"[10-12]. In graphs of Figs. 1 a), b) and 2) the behavior of the dark material collapsed into a black hole can be observed, meanwhile in Figs. 3 a), b) and 4 the behavior of black holes in process of collapse through emission of its particles towards its surroundings from its event horizon through the measurement of the rate of change of the entropy with respect to time and the different energy forms that can exist is shown. The rate in which the material reduces its rhythm of expansion, with 
the corresponding implications of heat transfer, is directly proportional to its contraction: probably "it is contracted in about thousands of millions of years" [10-21], if the density of other present dark materials in the universe does not contribute sufficiently to reduce that time.

\begin{tabular}{|c|c|c|c|c|}
\hline Fig. & $\begin{array}{c}\rho \text { o } S \text { graphication } \\
\text { rank }\end{array}$ & $\begin{array}{l}\text { Graphing rank of } \\
\text { real numbers } \\
(\operatorname{Re}(S), \operatorname{Re}(\rho))\end{array}$ & $\begin{array}{l}\text { Graphing rank of } \\
\text { imaginary numbers } \\
(\operatorname{Im}(S), \operatorname{Im}(\rho))\end{array}$ & $\begin{array}{c}\text { Real rank / } \\
\text { Imaginary rank for } \\
\text { the observer }\end{array}$ \\
\hline 1 & $-9^{9^{9}}$ at $9^{9^{9}}$ & $\begin{array}{c}1.063852918 \text { at } \\
1.063852922\end{array}$ & $\begin{array}{c}2.581342270 \text { at } \\
2.581342278\end{array}$ & $\begin{array}{l}4.0 \times 10^{-9} / \\
8.0 \times 10^{-9}\end{array}$ \\
\hline 2 & $\begin{array}{c}-157389592 \text { at } \\
157389592\end{array}$ & $\begin{array}{l}1.063852913 \text { at } \\
1.063852923\end{array}$ & $\begin{array}{l}-2.335331 \text { at } \\
-2.335324\end{array}$ & $\begin{array}{l}1.0 \times 10^{-8} / \\
7.0 \times 10^{-6}\end{array}$ \\
\hline 3 & $\begin{array}{c}-157389592 \text { at } \\
157389592\end{array}$ & -0.6 at -0.1 & $\begin{array}{c}3.39060 \times 10^{7} \text { at } \\
3.39069 \times 10^{7}\end{array}$ & $0.5 / 900$ \\
\hline 4 & $\begin{array}{c}0- \\
3.325585479 \times 10^{30} I \\
\text { at } \\
4.743179992 \times 10^{-15} \\
-8.744121208 \times 10^{-1}\end{array}$ & $\begin{array}{c}0 \\
\text { at } 4.743179992 \times 10^{-15}\end{array}$ & $\begin{array}{c}3.325585479 \times 10^{30} I \mathrm{a} \\
\mathrm{t} \\
-8.744121208 \times 10^{-16} I\end{array}$ & $\begin{array}{l}4.743179992 \times 10^{-15} / \\
\approx 3.325585479 \times 10^{30} I\end{array}$ \\
\hline
\end{tabular}

The curves bent upwards in Fig. 4, indicate that black material is contracted or it is in a collapsed state. Fig. 2 is an analogy of Fig. 1 graph in a different rank, for the same material is obtained more information in Fig. 2 what probably changes its history. Figs. 1 b) and 2 b) shows that the energy of the collapsed material into a black hole is quantized (points of the graph whose abscissa is the real component and its ordinate the imaginary component). The developed model and the simulated ranks in Figs. 1-4 do not allow to determine with exactitude the speed of a particle that falls in the black hole, nevertheless, according to the behavior observed in Fig. 3 a) and b), it is possible to argue that the particle exceeds the speed of light, which places it in a position outside the black hole to being "emitted" to the surroundings. This heat transfer measured through change of the hole entropy, $\partial S / \partial t$, is responsible of the disappearance of the black hole in the space-time and the new histories to add about its particles as members of "new families" suspended in the universe.

Here also it is possible to argue that the dark material simulated in Fig. 1 and 2 can correspond to the history of a baby universe where the material expulsed from the hole was confined. In Figs. $1 \mathrm{~b}$ ) and $2 \mathrm{~b}$ ) the points of the graphs can be associated with the sum of the probabilities of stories of all particles: "like pass through certain points in certain moments" predicted by Richard Feynman [22, 23]. Similarly, the sum of Feynman's stories can be related to the different curved space-time from Fig 4. The aligned points in vertical form in Figs. 1 b) and 2 b) indicate beginning and end of a black hole as if they were North and South latitudes in the globe.

The graph in Fig. 4 can be compared with a very compressed region of huge density or a fundamental black hole with an approximated mass of a mountain, in which, the quantum effect conceive it with a $10^{-15}$ radius centimeters turning in orbit around the Sun or the center of a galaxy (teorem " a black hole has no hair" [14-21], see axle of the real numbers). The degree of disorder observed in Fig. $3 \mathrm{~b}$ ) is related to a law that determines that the change of mass of a black hole is proportional to the area of its event horizon due to the gravity force on it [8]. 


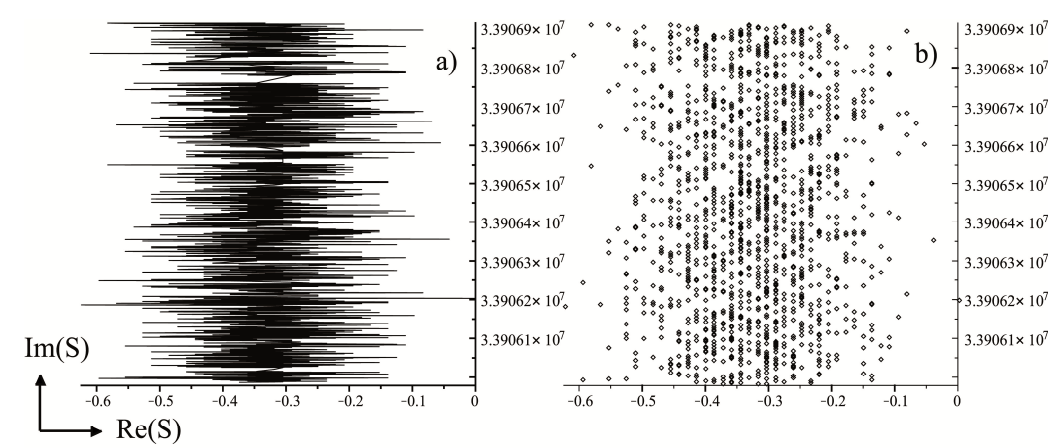

Fig. 3 Rate change of entropy, $\partial S / \partial t$, of a gigantic mass confined to a black hole. a) emissions of dark material since event horizon. b) Degree of disorder.

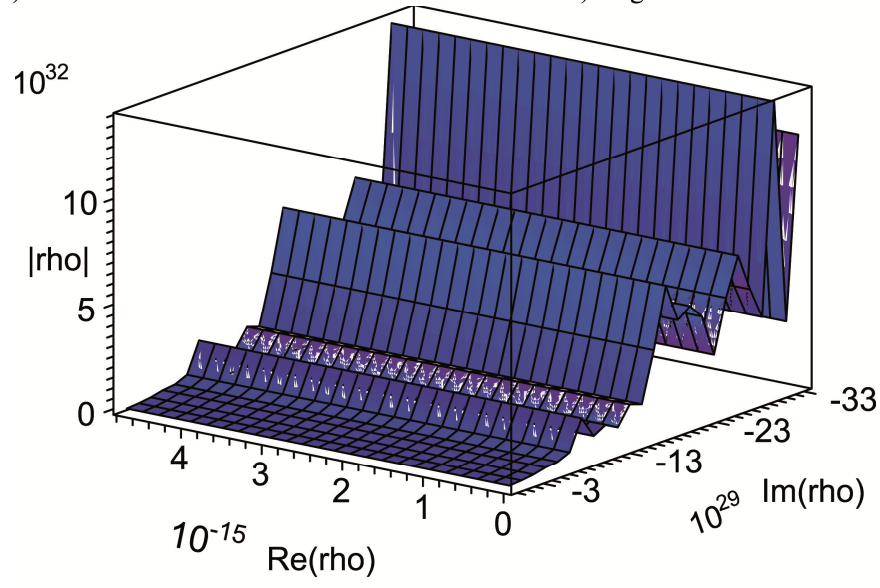

Fig. 4 Rate reduction of expansion $(\partial \rho / \partial t)$ of dark material of a black hole. The upward concavity indicates contraction in the hole.

\section{Conclusions}

From the modeling made to predict the behavior of gigantic masses in the space-time continuum by means of relativistic equations, a solution in imaginary terms is obtained that allow to argue evidence of: existence of dark material that constitutes a black hole; particle histories with certain properties that are developed in imaginary time; constitutive material of the constituent material of Hawking's "baby universes"; dark material quantized and colapsed into a black hole; a black hole in process of collapse through the emission of its particles towards the surroundings from its event horizonas a measure of its finite entropy, corroborated by Heisenberg's uncertainty principle that indicates that space-time finishes in the great collapse; heat transfer with a reduction rate proportional to the contraction of the hole; overlapping histories of particles with predictions of Feynman; and, sufficient reasons to satisface the theorem " a black hole has no hair".

\section{References}

[1] S. Hawking and R.Penrose: Proc. of the Royal Society Vol. 314 (1970), p. 529.

[2] K.Wark and D.E. Richards: Termodinámica, (Mc.Graw-Hill/Interamericana de España, S.A.U., Madrid, 2001).

[3] J.L. Tane: J. of Theor. Vol. 6 (6) (2004).

[4] J.H. Heinbockel: Introduction to Tensor Calculus and Continuum Mechanics, edited by J.H. Heinbockel (Old Dominion University, 1996), pp. 129-162.

[5] S. Chandrasekhar: Amer. J. Phys. Vol. 47 (3) (1979), p. 212.

[6] B. K. Datta and Bull Satyendranath Bose: Inst. Phys. Sci. Vol. 5 (2) (1980), p. 3.

[7] F. R. Hickman: Internat. J. Theoret. Phys. Vol. 23 (6) (1984), p. 535.

[8] P.T. Landsberg: Europ. J. Phys. Vol. 2 (4) (1981), p. 203. 
[9] J. Stachel: in: Conceptual problems of quantum gravity: Einstein and quantum mechanics, (Boston: MA, 1991), 13.

[10] S.W. Hawking: Black Holes and Baby Universes and Other Essays, (Bantam Books: London, 1994).

[11] S.W. Hawking: Comm. Math. Phys. Vol. 43 (3) (1975), p. 199.

[12] S.W. Hawking: Comm. Math. Phys. Vol. 46 (2) (1976), p. 206.

[13] S.W. Hawking: Nature Vol. 248 (1) (1974), p. 30.

[14] S.W.Hawking: Phys. Rev. D Vol. 72084013 (2005).

[15] J.D. Bekenstein and M. Schiffer: Int. J. Modern Physics Vol. 1 (1990), p. 355.

[16] J. D. Bekenstein: Phys. Rev. D Vol. 30 (1984), p. 1669.

[17] J.D. Bekenstein: Phys. Rev. D Vol. 27 (10) (1983), p. 2262.

[18] J.D. Bekenstein: Phys. Rev. D Vol. 23 (1981), p. 287.

[19] J.D. Bekenstein: Phys. Rev. Lett. Vol. 46 (1981), p. 623.

[20] J.D. Bekenstein: Physics Today, (1980), p. 24.

[21] J.D. Bekenstein: Phys. Rev. D Vol. 9 (1974), p. 3292.

[22] R. Feynman, R. Leighton and M. Sands: The Feynman Lectures on Physics, edited by Addison-Wesley (2006).

[23] R.P. Feynman: Rev. Mod. Phys. Vol. 20 (1948), p. 367. 